Cell-Type-Specific Genome-wide Expression Profiling after Laser Capture Microdissection of Living Tissue

F. Marchetti, C. F. Manohar

February 11, 2005 
This document was prepared as an account of work sponsored by an agency of the United States Government. Neither the United States Government nor the University of California nor any of their employees, makes any warranty, express or implied, or assumes any legal liability or responsibility for the accuracy, completeness, or usefulness of any information, apparatus, product, or process disclosed, or represents that its use would not infringe privately owned rights. Reference herein to any specific commercial product, process, or service by trade name, trademark, manufacturer, or otherwise, does not necessarily constitute or imply its endorsement, recommendation, or favoring by the United States Government or the University of California. The views and opinions of authors expressed herein do not necessarily state or reflect those of the United States Government or the University of California, and shall not be used for advertising or product endorsement purposes.

This work was performed under the auspices of the U.S. Department of Energy by University of California, Lawrence Livermore National Laboratory under Contract W-7405-Eng-48. 


\title{
04-FS-021
}

\section{Cell-Type-Specific Genome-wide Expression Profiling after Laser Capture Microdissection of Living Tissue}

\author{
Final Report \\ Co-PI: Francesco Marchetti, Chitra F. Manohar \\ Biosciences Directorate
}

This work was performed under the auspices of the U.S. Department of Energy (DOE) by the University of California, Lawrence Livermore National Laboratory (LLNL) under Contract No. W-7405-Eng-48. The project 04-FS-021 was funded by the Laboratory Directed Research and Development Program at LLNL. 


\section{Project description}

The purpose of this technical feasibility study was to develop and evaluate robust microgenomic tools for investigations of genome-wide expression of very small numbers of cells isolated from whole tissue sections. Tissues contain large numbers of cell-types that play varied roles in organ function and responses to endogenous and exogenous toxicants whether bacterial, viral, chemical or radiation. Expression studies of whole tissue biopsy are severely limited because heterogenous cell-types result in an averaging of molecular signals masking subtle but important changes in gene expression in any one cell type(s) or group of cells. Accurate gene expression analysis requires the study of specific cell types in their tissue environment but without contamination from surrounding cells. Laser capture microdissection (LCM) is a new technology to isolate morphologically distinct cells from tissue sections. Alternative methods are available for isolating single cells but not yet for their reliable genome-wide expression analyses. The tasks of this feasibility project were to:

1. Develop efficient protocols for laser capture microdissection of cells from tissues identified by antibody label, or morphological stain.

2. Develop reproducible gene-transcript analyses techniques for single cell-types and determine the numbers of cells needed for reliable genome-wide analyses.

3. Validate the technology for epithelial and endothelial cells isolated from the gastrointestinal tract of mice

\section{Progress Report}

During the review of this feasibility study, it was suggested that, while setting up the protocols for microdissecting small number of cells from tissue sections, work be conducted with cell lines to develop methods for obtaining high quality RNA for genome-wide expression studies from small number of cells. Therefore, in the three and half months of this project we focused our effort on the two tasks of (a) developing small sample genetranscript analysis protocols to define genome-wide expression profiles of isolated cells and (b) using a newly acquired laser-capture microdissection (LCM) system to identify and isolate target cells from tissue sections using histochemical labeling while retaining cellular messenger RNA integrity.

\section{Isolation of $m R N A$ from small number of cells}

Human fibroblasts NHF-1 were grown under standard culturing conditions. Initially, cell pellets containing 10,000, 5,000 and 2,500 cells were used. RNA was isolated using the Pico Pure Kit (Arcturus) and the amount and quality of recovered RNA was analyzed using the NanoDrop spectrophotometer (NanoDrop Inc.,) and microchannel electrophoresis on the Agilent Lab-on-a-chip Bioanalyzer. We were successful in isolating sufficient quantities of fairly good quality total RNA. The criteria for quality was the absorbance at 260 and 280 $\mathrm{nms}$ and integrity was assessed by the $28 \mathrm{~S} / 18 \mathrm{~S}$ ribosomal RNA ratios.

Another dilution series was performed in duplicate and included samples from 10,000 cells down to 500 and 250 cells. There were two pellets for each dilution. RNA yield was good down to the sample with 1,000 cells in one series and using $500(47.86 \mathrm{pg} / \mu \mathrm{l})$ and 250 
$(44.09 \mathrm{pg} / \mu \mathrm{l})$ cell samples. Thus we were successful in isolating intact total RNA from a number of cells that can be easily microdissected from tissue sections. Figure 1 shows an example of the analyses of RNA by microchannel electrophoresis using samples serially diluted and ranging from nanograms to picograms.

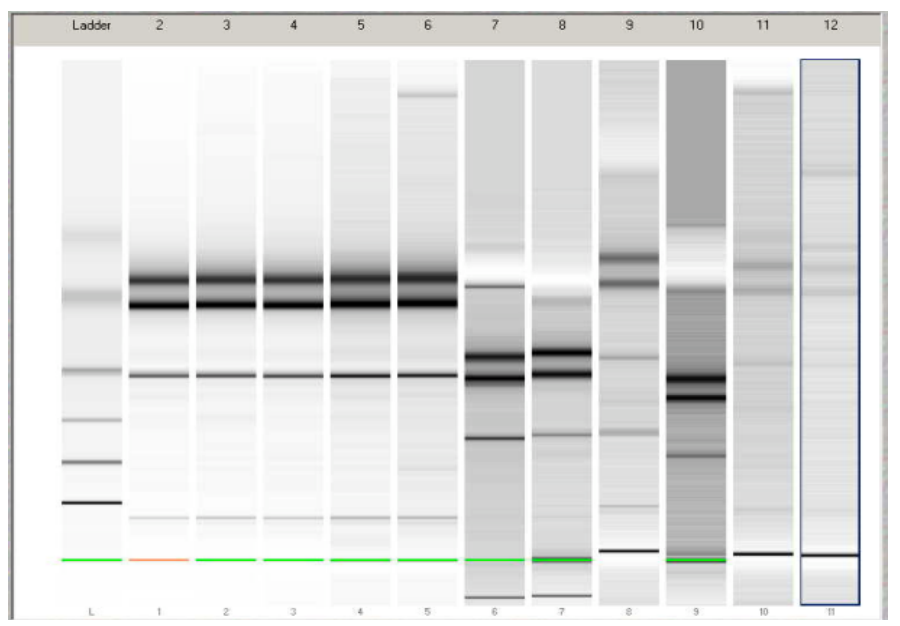

Figure 1: A dilution series of total RNA ranging from 4 nanograms to picograms (1-11 lanes; 4ng, 4ng, 2ng, 2ng, 1ng, $1 \mathrm{ng}, \quad 0.5 \mathrm{ng}, \quad 0.5 \mathrm{ng}, \quad .25 \mathrm{ng}$, $0.125 \mathrm{ng}, 62.5 \mathrm{ng}$ ) was analyzed on a PicoChip by Microchannel electrophoresis on the Agilent Bioanalyzer 2100.

We have also worked on small sample gene labeling protocols for DNA microarray and RT-PCR studies. Nanogram quantities (50-100ng) of total RNA were used to reverse transcribe RNA using T7 based oligodT primer and reverse transcriptase (Agilent Tech.) Double stranded cDNA was synthesisized followed by in vitro transcription using T7 RNA polymerase and Cy-3 and Cy-5 fluor-labelled NTPs. Labeled RNA was quantified using NanoDrop spectrophotometer. Microgram quantities of labeled linearly amplified RNA was generated and hybridized to in-house printed mouse partial genome arrays on glass slides with gamma aminosilane surface chemistry. Slides were hybridized in the Gene Machine HYB station, washed and scanned. We were successful in labeling nanogram quantitites of total RNA and label to high efficiency for microarray studies. In future, we plan to extend this method to label total RNA obtained from LCM derived tissues comprising of 5-10 nanograms.

\section{Preparation of tissue sections for microdissection}

As protocols for isolating RNA from small number of cells were being developed, work was conducted on preparing tissue sections for microdissection. Small intestine, brain and testis were isolated from B6C3F1 mice, immediately embedded in OTC compound. All three tissues were embedded on the same block and $10 \mu \mathrm{m}$ thick sections were cut using a microtome and put on positively-charged microscope slides. Slides were dehydrated with an ethanol series $(75 \%, 95 \%$ and $100 \%)$ and stained with a HistoGene staining solution (Arcturus) according to the manufacturer's specifications. The Arcturus' laser capture microdissection system AutoPix ${ }^{\mathrm{TM}}$ was used. The system automatically places microdissection caps a few microns above the area on the tissue selected for microdissection by the researcher. When the laser is fired, it melts a special polymer on the surface of the cap; the melted polymer drops until it touches the tissue and when the cap is removed, the area of the tissue in contact with the polymer is removed too. 
Initial microdissection attempts did not succeed. Although some tissue was microdissected, there was considerable variation in the tissue captures even within the same tissue region, therefore, new sections were prepared. This time each tissue was embedded in different OTC block and the thickness of the section was reduced to $8 \mu \mathrm{m}$. Fixation time in
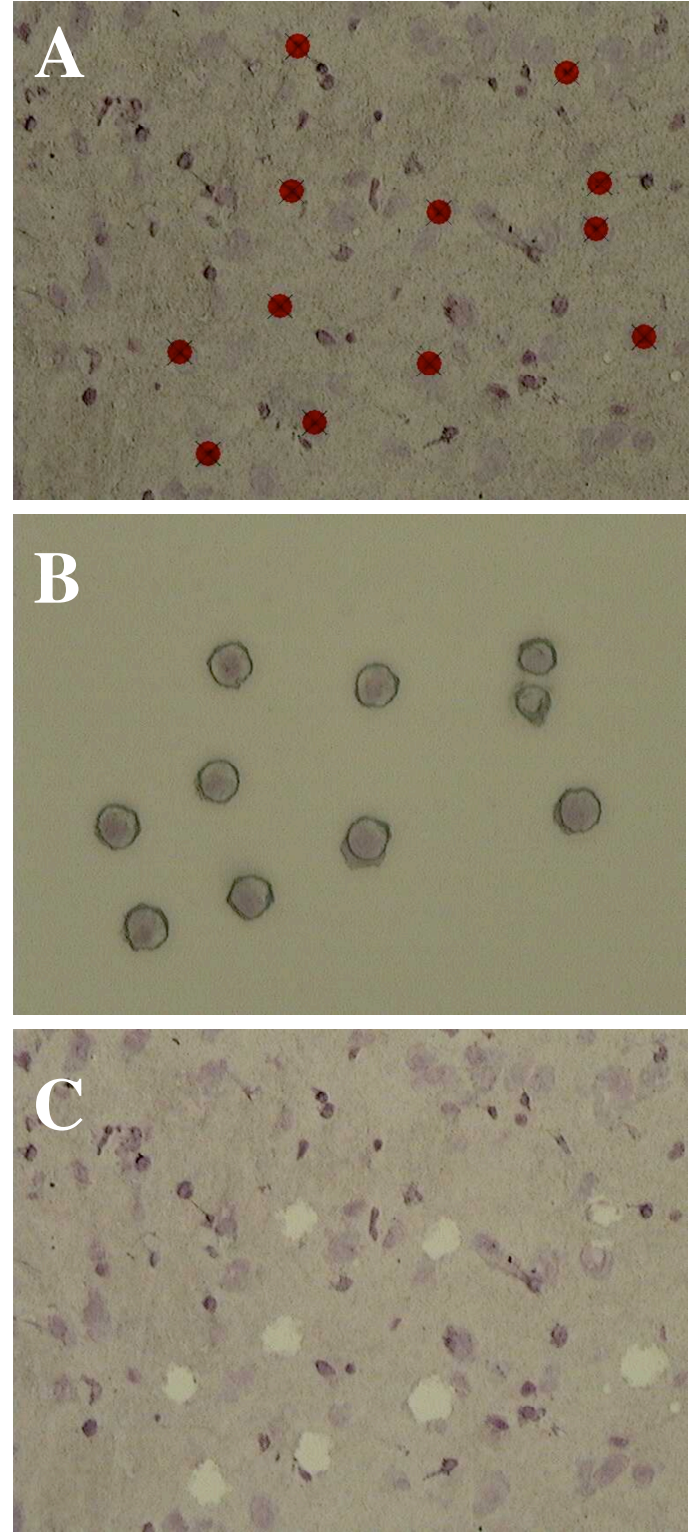

Figure 2 - Tissue microdissection performed using the LCM system. A. Section of mouse brain stained with Histogene staining solution. The red dots represent the neuron cells that were selected for microdissection by the researcher. B. Image of the surface of the microdissection cap showing the cells that were dissected from the tissue section. C. Image of the same in tissue section in A, showing empty spaces were the cells were microdissected from. xylene and ethanol was increased to remove any trace of water from the tissue section, which can affect capturing. Occasional good captures were obtained, however, there was still significant variation even within the same tissues. Additionally, the quality of RNA that was recovered from those tissue sections that were successfully microdissected was not good and showed signs of degradation.

We then performed a series of experiments to test parameters such as medium for embedding tissues, thickness of the sections (6 and $5 \mu \mathrm{m}$ sections were tried), staining procedure on the quality of microdissection and of RNA. We also contacted Arcturus for advice. Longer time at $-70^{\circ} \mathrm{C}$ before tissue fixation, extended time in ethanol and the use of plain microscope slides were among the suggestions provided. It was also discovered a manufacturing problem with a lot of the dissection caps that were being used, which were immediately replaced by Arcturus. New tissue sections were prepared following the above modifications and the quality of microdissection significantly improved. Figure 2 shows a mouse brain tissue section before (A) and after microdissection (C) and the cap with the microdissected cells (B). It can be seen that several single cells were successfully isolated. We also tested for RNA quality and the results were good. However, there were still some inconsistencies in tissue capturing among the various tissues, which could not be addressed before the end of funding.

\section{Summary}

During the three and half month period of this feasibility project we have successfully isolated total RNA from small number of cells. Beginning with samples containing 10,000 cells 
we have progressively reduced the number of cells used for isolating RNA down to 250 cells. Good results were consistently obtained with as few as 1000 cells, and, on one occasion we obtained significant amounts of good quality RNA from as few as 250 cells. Small sample gene labeling protocols for DNA microarray and RT-PCR studies have been developed. Methodology was developed for preparing sections from various mouse tissues (i.e., small intestine, kidney and testis) for LCM. We performed experiments to tests parameters such as medium for embedding tissues, thickness of the sections, staining procedure on the quality of RNA produced from microdissected cells. We successfully microdissected cells from tissues and isolated RNA, however, further work is needed to refine tissue preparation protocols to consistently obtain high quality RNA. 